\title{
The role of echocardiography in SARS-CoV-2 pandemic: a compromise among appropriateness, safety and clinical impact
}

\author{
Mario Pacileo $^{1}$, Francesco Giallauria ${ }^{2}$, Cristina Savarese ${ }^{1}$, Teresa Cirillo ${ }^{1}$, Fabio Crescibene ${ }^{3}$, \\ Anna Di Lorenzo ${ }^{2}$, Mariacarla Ferrillo', Maria Grazia Calabrese ${ }^{1}$, Carlo Vigorito², Antonello D'Andrea ${ }^{1,4}$ \\ ${ }^{1}$ Division of Cardiology, Hospital Umberto I, Nocera Inferiore (SA); ${ }^{2}$ Department of Translational Medical Sciences, \\ Federico II University of Naples; ${ }^{3}$ Division of Cardiology, COVID Hospital M. Scarlato, Scafati (SA); ${ }^{4}$ Division of \\ Cardiology, University of Campania L. Vanvitelli, Naples, Italy
}

\begin{abstract}
SARS-CoV-2 infection, responsible for COVID-19, can determine cardiac events, which require a quick diagnosis and management, and should not be overlooked due to the presence of COVID-19 infection. In some cases, cardiovascular symptoms can also be the first and only manifestation of SARS-CoV-2 infection. In patients with COVID-19, the full cardiovascular disease diagnostic algorithm can be hindered by logistic restrain mainly derived from the difficulty of transporting patients in critical conditions to Radiology or Hemodynamics wards. The echocardiog-
\end{abstract}

Correspondence: Mario Pacileo, Division of Cardiology/UTIC, Hospital Umberto I, Via San Francesco 65, 84014 Nocera Inferiore (SA), Italy.

Tel. +39.081.927570. E-mail: m.pacileo@aslsalerno.it

Key words: COVID-19; echocardiography; transesophageal echocardiography; ARDS; PPE.

Contributions: MP, ADA, contributed to the conception of the work; MP, ADA, FG, FC, drafted the manuscript; CS, TC, ADL, MGC, MF, $\mathrm{CV}$, contributed to the acquisition, analysis, and interpretation of data for the work; MP, ADA, FG, CV, critically revised the manuscript. All authors gave final approval and agree to be accountable for all aspects of work ensuring integrity and accuracy.

Conflict of interests: All authors declare that they have no known competing financial interests or personal relationships that could have appeared to influence the work reported in this paper.

Acknowledgements: Authors are grateful to Filomena Aufiero, Giuseppina Fiume and Renato De Luca for excellent nursing and rehabilitation support in our cardiac patients.

Received for publication: 3 May 2020

Accepted for publication: 13 May 2020.

${ }^{\circ}$ Copyright: the Author(s), 2020

Licensee PAGEPress, Italy

Monaldi Archives for Chest Disease 2020; 90:1358

doi: 10.4081/monaldi.2020.1358

This article is distributed under the terms of the Creative Commons Attribution Noncommercial License (by-nc 4.0) which permits any noncommercial use, distribution, and reproduction in any medium, provided the original author(s) and source are credited. raphy in SARS-CoV-2 pandemic can help for differential diagnosis of cardiac events, which can be related or unrelated by the infection and can likely impact on short-term prognosis. Indeed, transthoracic echocardiography plays a key role in the screen for CV complications of COVID-19 infection: it must be focused cardiac ultrasound study (FoCUS) performed at bedside. All transthoracic, transesophageal and stress echocardiograms in patients in which test results are unlikely to change the management strategy should be postponed.

In these weeks of great national emergency we have learned that SARS-CoV-2, the virus responsible for COVID-19, can determine cardiac events, which can be related to the infection including acute coronary syndromes (STEMI and NSTEMI), acute heart failure, arrhythmias, myocarditis, pericarditis, thromboembolic events, cardiogenic shock, and cardiac arrest. Those syndromes require a quick diagnosis and management, and should not be overlooked due to the presence of COVID-19.

Cough, fever, tiredness and difficulty breathing compose the "classic" COVID-19 symptoms; some SARS-CoV-2 positive patients have anosmia and dysgeusia and gastrointestinal disturbances. Moreover, these patients often show symptoms mimicking cardiovascular events, including chest pain, dyspnea, and shock, even in the absence of cardiac injury. In some cases, cardiovascular symptoms can also be the first and only manifestation of SARS-CoV-2 infection [1,2].

Some positive patients are admitted to the emergency room with palpitations and tachycardia, chest tightness or pain, and drop attack or syncope. These disorders are caused by arrhythmias, acute myocardial infarction, acute heart failure, myocarditis and pericarditis. Indeed, associated profound hypoxemia related underlying pneumonia together with tachycardia might result in chest pain and electrocardiographic changes suggestive of myocardial ischemia. The prevalence of cardiac injury is estimated up to $20 \%$ of cases. The severity of cardiac involvement is closely related to the severity of clinical scenario and mortality in these patients [3-7].

The appearance of acute myocardial injury is not early but it usually occurs at least 10 days after the appearance of fever $[5,8]$. Cardiomyocyte injury, as quantified by cardiac troponin T/I concentrations, and hemodynamic stress, as quantified by B-type natriuretic peptide (BNP) and N-terminal B type natriuretic peptide (NT-proBNP) concentrations, may occur in as in COVID-19 infections as in other pneumonias. The level of those biomarkers 
correlated with disease severity and mortality $[5,6]$. Concentrations of these biomarkers remained within the normal range in the majority of survivors; however, in non-survivors, troponin levels progressively increased in parallel with the severity of COVID-19 and ARDS development $[6,9,10]$.

During the acute phase, the occurrence of overall reduction of systolic function of the left ventricle can be assessed by echocardiography, in the absence of previous history of systolic dysfunction cardiomyopathy, especially in patients with clinical signs of cardiogenic shock admitted to Intensive Care Unit [11].These features are usually seen in other conditions characterized by cytokine-mediated responses such as septic shock, where the release of cytokines can determine reduction of the ejection fraction and increase in the volume of the chambers ventricles [12]; and in acute myocardial infarction, where the cytokine response contributes to the mechanisms of cardiogenic shock [13]. Therefore, studies aiming at clarifying the peculiarity of this type of cardiac impairment during COVID-19 are eagerly encouraged.

Myocarditis and pericarditis are other manifestations of cardiac involvement from SARS-CoV-2 occurring sometimes before the onset of pulmonary symptoms shock [14-16]. In addition, myocardial injury with ST-segment elevation on electrocardiography has been observed in patients with Covid-19. These patients have variability in presentation, a high prevalence of non-obstructive disease, and a poor prognosis shock [16-18]. It has been reported the presence of SARS-CoV-2 in the pericardial fluid of a patient with COVID19 and cardiac tamponade subjected to pericardiocentesis shock [19].

The mechanism underlying acute myocardial injury is not fully elucidated; however, a direct cardiac injury could be hypothesized. In some cases, the SARS-CoV-2 can cause direct damage to myocytes mediated by stimulation of the angiotensin-converting enzyme 2 (ACE2), which is expressed on myocytes and vascular endothelial cells, acting as a receptor for SARS-CoV-2 and as "gateway" for the virus in these cells. Another hypothesized mechanism is myocardial damage induced by hypoxia and activation of the innate immune response with release of pro-inflammatory cytokines: a real inflammatory storm, as well as to the activation of adaptive auto-immune which can induce vascular and myocardial inflammation and an excess of blood clotting, which cause episodes of diffuse thrombosis shock [9].

In patients with COVID-19, the full cardiovascular disease diagnostic algorithm can be hindered by logistic restrain mainly derived from the difficulty of transporting patients in critical conditions to Radiology or Haemodynamics wards. Therefore, Transthoracic (TT) Echocardiography shock [18] plays a key role in the initial diagnostic phase, since it can be performed bedside. However, it should be considered that in COVID-19 patients, echocardiography should be carried out according to the guidance provided by scientific societies shock [19], limiting the exposure time of the operators at the acquisition of images strictly necessaries (Table 1). Focused cardiac ultrasound study (FoCUS) performed at bedside is an effective option to screen for CV complications of COVID-19 infection. It should not be forgotten that the risk of infection remains in the reading rooms and therefore the device should be also frequently sanitized. Finally, the quality of echocardiography can be compromised in intubated patients shock (Figure 1) [18-22].

Non-urgent or elective cardiac imaging should not be performed routinely in patients with suspected or confirmed COVID19 infection and should be postponed until the infection has terminated [21]. All transthoracic, transesophageal and stress echocardiograms in patients in which test results are unlikely to change the management strategy should be postponed.

In this clinical scenario, echocardiography is useful for evaluating regional or global anomalies of the contractility of the left ventricle, and documenting the possible acute overload of the right ventricle, in case of ARDS or pulmonary embolism, especially in presence of hemodynamic instability [20].

A single ultrasound system should be identified, preferably portable and with reduced dimensions to facilitate sanitation; probe covers should be used, and a single operator with sufficient experience (not in training) should perform the examination. The final exam scan should be a good compromise between adequacy and speed of execution (limited ultrasound sections, bimodal responses with high clinical impact, image registration and subsequent "off line" review).

Table 1. Schematic representation of echocardiographic parameters for the assessment of COVID-19 patients and suspected cardiovascular involvement.

\begin{tabular}{lll} 
Cardiac chamber & Clinical question & What to analyze \\
Left ventricle & Septic shock & Increased LV size (diameters or volumes) \\
& Significant troponin increase & Reduced LV ejection fraction \\
& Suspected myocarditis & Impaired LV Segmental contractile function \\
Left atrium & Supraventricular arrhythmias & Increased LA size (diameter or volume) \\
\hline Right ventricle & Pulmonary embolismInvasive or noninvasive ventilation with & Increased RV size (diameters) \\
& unstable hemodynamics & Dilated RV with basal RV/LV ratio $>1.0$, and McConnell sign \\
& & Decreased tricuspid annular plane systolic excursion (TAPSE) \\
Inferior vena cava & Hemodynamic impairment & Pulmonary hypertension (increased PASP) \\
\hline Pericardium & Pericarditis & Distended IVC with reduced inspiratory collapsibility \\
& Suspected cardiac tamponade & Pericardial effusion \\
& & Right heart chamber collapse
\end{tabular}

$\mathrm{LV}$, left ventricle; RV, right ventricle; PASP, pulmonary artery systolic pressure; IVC, inferior vena cava. 
Particular attention should be paid to the execution of transesophageal echocardiogram (TEE). TEE carries increased risks of spread of COVID-19 due to exposure to aerosol of large viral load and it should not be performed if an alternative imaging modality is available. TEE must be carried out with complete personal protective equipment (i.e., mask at least N95 according to American classification or FFP2 according to the European classification).

To date, studies focused on Doppler-echocardiography parameters conducted specifically in cohorts of COVID-19 patients are lacking. However, the role of echocardiography in monitoring biventricular function during sepsis and septic shock is well known. Most studies on acute myocardial dysfunction during sepsis have been conducted in patients admitted to intensive care and subjected to mechanical ventilation, using TT and TEE echocardiography [24,25]. These studies show that about $30-40 \%$ of septic patients develop reduction of the ejection fraction of the left ventricle, impaired diastolic function of the left ventricle and possible impairment of the function of the right sections in particular during ARDS [26,27].

Another aspect that should be underlined is the hemodynamic effect of ventilation on cardiac performance [29,29]. Positive endexpiratory pressure (PEEP), increasing lung size, can cause an increase in pulmonary vascular resistance (RVP), due to the com- pression of alveolar and extra-alveolar capillaries; as a consequence, there is increase in post-load and right ventricle volume (VD), shift of the interventricular septum to the left and reduction of compliance and filling of the left ventricle. However, since the increase in RVP is countered by the elimination of hypoxiainduced vasoconstriction, increase in RVP becomes relevant only for high PEEP values. In addition, the increase in intrathoracic pressure determines a subsequential increase in intrathoracic veins resistance, that represents a mechanism reducing the gradient and venous return for VD, especially in conditions of true (anemia, dehydration, etc., in a healthy heart) or relative (severe right ventricular dysfunction) hypovolemia. This phenomenon, however, is counterbalanced by the increase in abdominal pressure due to the diaphragm lowering with the effect of live and spleen "squeezing". Therefore, in clinical practice, the potential negative hemodynamic effects of PEEP (hypotension secondary to reduced preload and increased VD afterload) are mainly confined to patients highly dependent on preload, and can be partially balanced by carrying out a preventive volemic filling (Figure 2) [29-31].

Therefore, in this particular period, we have to be careful to possible cardiological symptoms of COVID-19. The echocardiography in SARS-CoV-2 pandemic can help for differential diagnosis of cardiac events, that can be related or unrelated by the infection but can be determinants for the short-term prognosis.

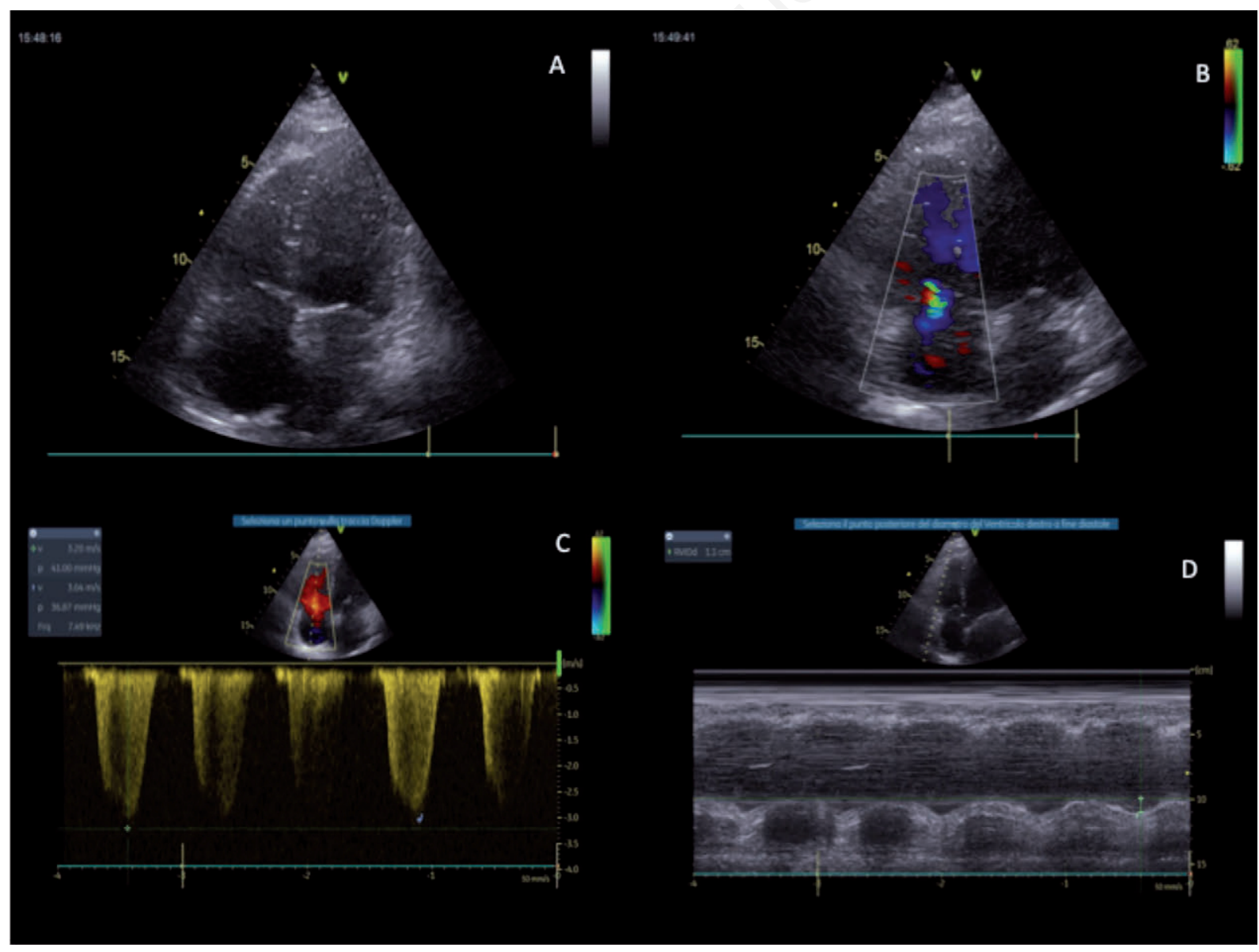

Figure 1. 65-year-old male patient, affected by COVID pneumonia, intubated, increased levels of troponin and D-Dimers, and negative CUS. Normal size of the left ventricle and normal ejection fraction, mild dilation of the right ventricle (A), mild tricuspid regurgitation (B), increased PAPs (C) and severe contractile dysfunction of the right ventricle, with reduced tricuspid annular plane systolic excursion (TAPSE) (D). 


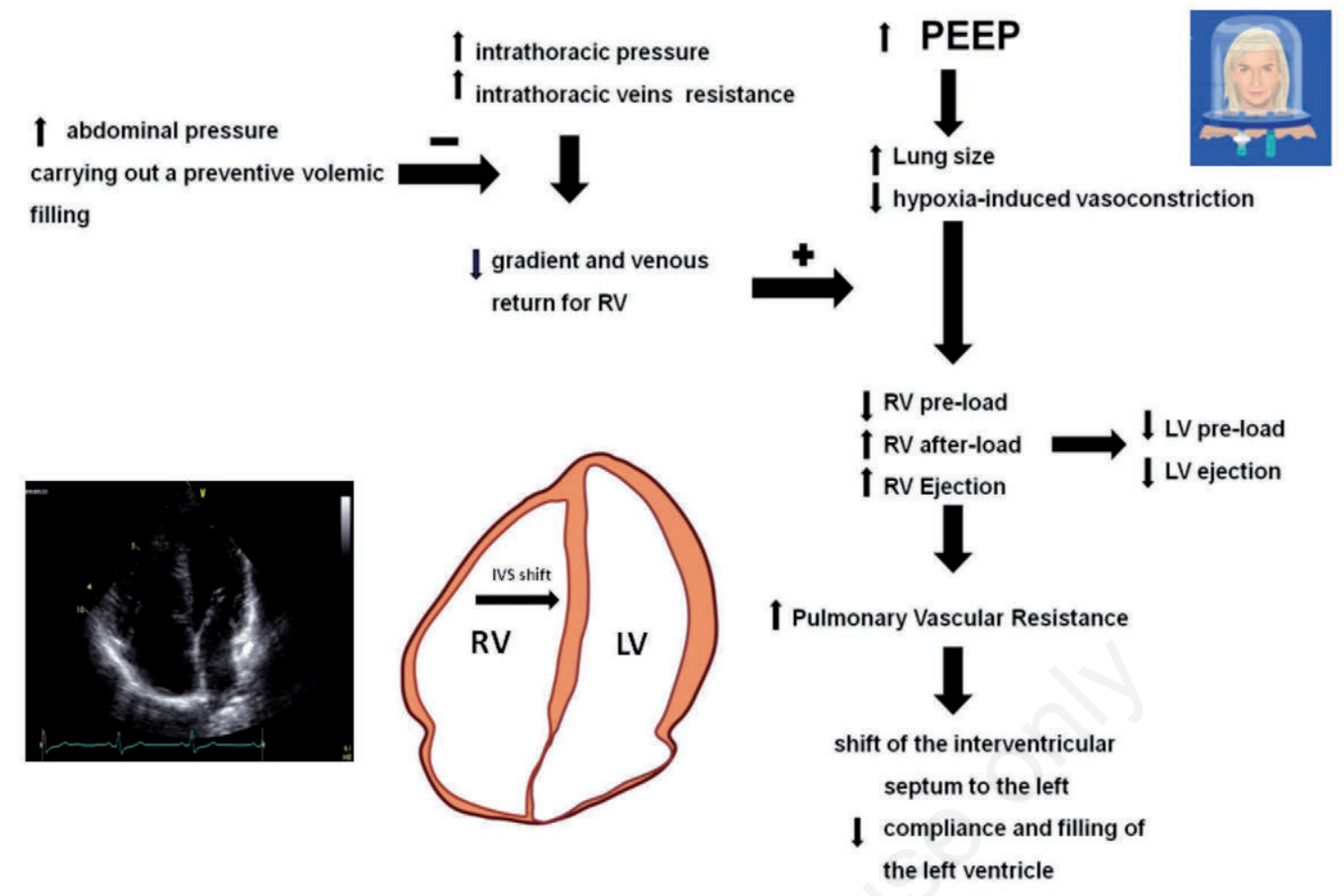

Figure 2. Hemodynamic effects of ventilation on cardiac performance. PEEP, positive end-expiratory pressure; RV, right ventricle. LV, left ventricle; IVS, interventricular septum.

\section{References}

1. Xiong T-Y, Redwood S, Prendergast B, et al. Coronaviruses and the cardiovascular system: acute and long-term implications. Eur Heart J 2020. doi:10.1093/eurheartj/ehaa231.

2. Madjid M, Safavi-Naeini P, Solomon SD, et al. Potential Effects of Coronaviruses on the Cardiovascular System. A Review. JAMA Cardiol 2020. doi:10.1001/jamacardio.2020.1286.

3. Huang C, Wang Y, Li X, et al. Clinical features of patients infected with 2019 novel coronavirus in Wuhan, China. Lancet 2020;395:497-506.

4. Wang D, Hu B, Hu C, et al. Clinical characteristics of 138 hospitalized patients with 2019 novel coronavirus-infected pneumonia in Wuhan, China. JAMA 2020. doi:10.1001/jama.2020.1585.

5. Zhou F, Yu T, Du R, et al. Clinical course and risk factors for mortality of adult inpatients with COVID-19 in Wuhan, China: a retrospective cohort study. Lancet 2020;395:1054-1062.

6. Shi S, Qin M, Shen B, et al. Association of cardiac injury with mortality in hospitalized patients with COVID-19 in Wuhan, China. JAMA Cardiol 2020. doi:10.1001/ jamacardio.2020. 0950.

7. Bonow RO, Fonarow GC, O'Gara PT, Yancy CW. Association of Coronavirus Disease 2019 (COVID-19) With Myocardial Injury and Mortality. JAMA Cardiol 2020. doi:10.1001/jamacardio.2020.1105.

8. Deng Q, Hu B, Zhang Y, et al. Suspected myocardial injury in patients with COVID-19: Evidence from front-line clinical observation in Wuhan, China. Int J Cardiol 2020, doi: 10.1016/j.ijcard.2020.03.087.
9. Zheng Y-Y, Ma Y-T, Zhang J-Y, Xie X. COVID-19 and the cardiovascular system. Nature Rev Cardiol2020;17:259-60. doi: 10.1038/s41569-020-0360-5.

10. Chapman AR, Bularga A, Mills NL. High-sensitivity cardiac troponin can be an ally in the fight against COVID-1. Circulation 2020. doi: 10.1161/CIRCULATIONAHA.120.047008

11. Arentz M, Yim E, Klaff L, et al. Characteristics and outcomes of 21 critically ill patients with COVID-19 in Washington State. JAMA 2020. doi:10.1001/jama.2020.4326.

12. Parrillo JE. Pathogenetic mechanism of septic shock. N Engl J Med 1993;328:1471-7.

13. Van Diepen S, Katz JN, Albert NM, et al. Contemporary management of cardiogenic shock: A scientific statement from the American Heart Association. Circulation 2017;136: e232-e68.

14. Hua A, O'Gallagher K, Sado D, Byrne J. Life-threatening cardiac tamponade complicating myo-pericarditis in COVID-19. Eur Heart J 2020. doi:10.1093/eurheartj/ehaa253.

15. Zeng J-H, Liu Y-X, Yuan J, et al. First case of COVID-19 complicated with fulminant myocarditis: a case report and insights. Infection 2020. doi: 10.1007/s15010-020-01424-5.

16. Inciardi RM, Lupi L, Zaccone G, et al. Cardiac involvement 1 with coronavirus 2019 (COVID-19) infection. JAMA Cardiol 2020. doi:10.1001/ jamacardio.2020.1096.

17. Bangalore S, Sharma A, Slotwiner A, et al. ST-segment elevation in patients with Covid-19 - A case series. N Engl J Med 2020. doi: 10.1056/NEJMc2009020.

18. Skulstad H, Cosyns B, Popescu BA, et al. COVID-19 pandemic and cardiac imaging. EACVI recommendations on precautions, indications, prioritisation and protection for patients and 
healthcare personnel. Eur Heart J Cardiovasc Imaging 2020. doi: 10.1093/ehjci/jeaa072.

19. Farina A, Uccello G, Spreafico M, et al. SARS-CoV-2 detection in the pericardial fluid of a patient with cardiac tamponade. Eur J Intern Med 2020 doi.org/10.1016/j.ejim.2020.04.045.

20. D'Andrea A, Di Giannuario G, Marrazzo G, et al. The role of multimodality imaging in COVID-19 patients: from diagnosis to clinical monitoring and prognosis. G Ital Cardiol 2020;21:345-53. doi: 10.1714/3343.33132.

21. Kirkpatrick J, Mitchell C, Taub C, et al. ASE statement on protection of patients and echocardiography service providers during the 2019 novel coronavirus outbreak. J Am Coll Cardiol 2020;S0735-1097(20)34815-4. doi: 10.1016/j.jacc.2020.04.002.

22. Tavazzi G, Pellegrini C, Maurelli M, et al. Myocardial localization of coronavirus in COVID-19 cardiogenic shock. Eur J Heart Fail 2020. doi: 10.1002/ejhf.1828.

23. Shi S, Quin M, Shen Bo et al. Association of cardiac injury with mortality in hospitalized patients with COVID-19 in Wuhan, China. JAMA Cardiol 2020. doi:10.1001/jamacardio.2020.0950.

24. Bouhemad B, Nicolas-Robin A, Arbelot C, et al. Acute left ventricular dilatation and shock-induced myocardial dysfunction. Crit Care Med 2009;37:441-7.

25. Vieillard-Baron A, Caille V, Charron C, et al. Actual incidence of global left ventricular hypokinesia in adult septic shock. Crit Care Med 2008;36:1701-6.

26. Furian T, Aguiar C, Prado K, et al. Ventricular dysfunction and dilation in severe sepsis and septic shock: Relation to endothelial function and mortality. J Crit Care 2012;27:319.e9-15. doi: 10.1016/j.jcrc.2011.06.017.

27. Parker MM, McCarthy KE, Ognibene FP, Parrillo JE. Right ventricular dysfunction and dilatation, similar to left ventricular changes, characterize the cardiac depression of septic shock in humans. Chest 1990;97:126-31.

28. Vagnarelli F, Marini M, Caretta G. [Ventilazione non invasiva: caratteri generali, indicazioni e revisione della letteratura]. [Article in Italian]. G Ital Cardiol 2017;18:496-504.

29. Luchetti M, Moretti C. [La ventilazione non invasiva e le sue implicazioni emodinamiche].[Article in Italian]. Acta Aneastesiologica Italica 2008;59:227-241.

30. D'Andrea A, Martone F, Liccardo B, et al. Acute and chronic effects of noninvasive ventilation on left and right myocardial function in patients with obstructive sleep apnea syndrome: A speckle tracking echocardiographic study. Echocardiography 2016;33:1144-55. doi: 10.1111/echo.13225.

31. Guarracino F. [Ecocardiografia in Area Critica].[Book in Italian]. Elsevier Srl, Milan. 